\title{
Tratamento cirúrgico conservador de ameloblastoma: relato de caso
}

Conservative surgical treatment of ameloblastoma: case report

Tratamiento quirúrgico conservador de ameloblastoma: reporte caso

Maylson Alves Nogueira BARROS ${ }^{1}$

Vitor Bruno TESLENCO ${ }^{1}$

Claudio Marcio SANTANA ${ }^{1}$

Lucas Marques MEURER ${ }^{1}$

Hebert de Abreu CAVALCANTI ${ }^{2}$

Guilherme Nucci REIS ${ }^{2}$

Everton Floriano PANCINI ${ }^{2}$

${ }^{I}$ Residente em Cirurgia e Traumatologia Bucomaxilofacial, Associação Beneficente da Santa Casa, 79002-251, Campo Grande - MS

${ }_{2}^{2}$ Preceptor do programa de residência em Cirurgia e Traumatologia Bucomaxilofacial, Associação Beneficente da Santa Casa, 79002-251, Campo Grande-MS.

\section{Resumo}

Introdução: $\mathrm{O}$ ameloblastoma é um tumor benigno com origem do epitélio odontogênico advindo dos restos da lâmina dentaria, revestimento de cistos ou epitélio basal da cavidade oral. Pode apresentar comportamento agressivo, invasivo, com crescimento lento associado a altas taxas de recidiva, transformação maligna e metástase. Variedades de abordagens são propostas para estes tumores, abordagens agressivas como ressecção com margem ou em bloco, até as mais conservadoras. Objetivo: Este relato de caso tem como objetivo expor para a comunidade acadêmica o tratamento cirúrgico conservador de um ameloblastoma. Relato de caso: Paciente leucoderma, 28 anos, sexo masculino, com queixa de aumento de volume na região submandibular há aproximadamente um ano, com dor e relatando dormência em lábio inferior. Apresenta imagem hipodensa envolvendo a mandíbula anterior e bilateral posterior, até a região dos segundos molares, provocando severa expansão óssea e reabsorção radicular apical. Foi realizada uma biopsia incisional onde foi confirmado diagnostico de ameloblastoma, subsequentemente foi submetido tratamento conservador. Conclusão: O tratamento proposto se demonstrou satisfatório até o momento, visto que obtivemos neoformação óssea e foi possível evitar mutilações.

Descritores: Ameloblastoma; Patologia Bucal; Neoplasias Bucais.

\section{Abstract}

Introduction: Ameloblastoma is a benign tumor with origin of the odontogenic epithelium coming from the remains of the dental blade, lining of cysts or basal epithelium of the oral cavity. It may present aggressive, invasive, slow-growing behavior associated with high rates of recurrence, malignant transformation and metastasis. Varieties of approaches are proposed for these tumors, aggressive approaches such as margin or block resection, even the most conservative ones. Objective: This case report aims to expose the conservative surgical treatment of an ameloblastoma to the academic community. Case report: Leucoderma patient, 28 years old, complaining of increased volume in the submandibular region for approximately one year, with pain and reported paresthesia in the lower lip. It presents a hypodense image involving the mandibular anterior and posterior bilateral, until the region of the second molars, causing severe bone expansion and apical root resorption. An incisional biopsy was performed in which the diagnosis of ameloblastoma was confirmed, and subsequently a conservative treatment. Conclusion: The proposed treatment proved to be satisfactory so far, since we obtained bone neoformation and it was possible to avoid mutilations

Descriptors: Ameloblastoma; Pathology, Oral; Mouth Neoplasms.

\section{Resumen}

Introduccíon: El ameloblastoma es un tumor benigno con origen del epitelio odontogénico proveniente de los restos de la lámina dental, revestimiento de quistes o epitelio basal de la cavidad oral. Puede presentar comportamiento agresivo, invasivo, con crecimiento lento asociado a altas tasas de recidiva, transformación maligna y metástasis. Variedades de abordajes son propuestas para estos tumores, abordajes agresivos como resección con margen o en bloque, hasta las más conservadoras. Objetivo: Este relato de caso tiene como objetivo exponer para la comunidad académica el tratamiento quirúrgico conservador de un ameloblastoma. Relato de caso: Paciente leucoderma, 28 años, con queja de aumento de volumen en la región submandibular hace aproximadamente un año, con dolor y relatando adormecimiento en labio inferior. Se presenta una imagen hipodensa envolviendo la mandíbula anterior y bilateral posterior, hasta la región de los segundos molares, provocando severa expansión ósea y reabsorción radicular apical. Se realizó una biopsia incisional donde se confirmó el diagnóstico de ameloblastoma, posteriormente fue sometido a tratamiento conservador. Conclusión: El tratamiento propuesto se demostró satisfactorio hasta el momento, ya que obtuvimos neoformación ósea y fue posible evitar mutilaciones.

Descriptores: Ameloblastoma; Patología Bucal; Neoplasias de la Boca.

\section{INTRODUÇÃO}

O ameloblastoma é um tumor benigno com origem do epitélio odontogênico advindo dos restos da lâmina dentaria, revestimento de cistos ou epitélio basal da cavidade oral. Pode apresentar comportamento agressivo, invasivo, com crescimento lento associado a altas taxas de recidiva, transformação maligna e metastase $e^{1,2}$. A patogênese do ameloblastoma é controversa, estudos sugerem ativação da MAPK (proteína quinase ativada por mitógeno), exerce função importante, agregada a estimulação do fator de necrose tumoral alfa $\left(\mathrm{TNF}-\alpha^{2,3}\right.$. São classificados e separados conforme apresentações clinicas, radiográficas e histológicas. São subdivididos em: Sólido convencional, unicistico e periférico ${ }^{1}$.

O ameloblastoma convencional tem predisposição de exibir alterações císticas, com combinações variadas de características. Múltiplos subtipos histopatológicos são conhecidos, sendo os mais comuns folicular e plexiforme, entre outros menos comuns o acantomatoso, de células granulares, desmoplásico e de células basais ${ }^{1,4}$.

Variedades de abordagens são propostas para estes tumores, abordagens agressivas como ressecção com margem ou em bloco, até as mais conservadoras como descompressão associadas a enucleação e curetagem ${ }^{1,4}$.

Este relato de caso tem como objetivo expor para a comunidade acadêmica o tratamento conservador de um ameloblastoma.

\section{CASO CLÍNICO}

Paciente leucoderma, 28 anos, encaminhado ao ambulatório do serviço de cirurgia e traumatologia bucomaxilofacial, com queixa de aumento de volume 
na região submandibular há aproximadamente um ano, com dor e relatando dormência em lábio inferior. Ao exame clinico apresentava aumento de volume na região mandibular anterior, observado em vista extraoral.

Ao exame tomográfico, foi observada severa lesão hipodensa com presença de septações, apresentando limites definidos, envolvendo a mandíbula anterior e bilateral posterior, até a região dos segundos molares, provocando severa expansão óssea e reabsorção radicular apical externa dos dentes $46,45,44,34,35$ e 36 . Conforme o laudo realizado pelo radiologista, a hipótese diagnóstica era de Granuloma central de células gigantes (Figuras 1, 2 e 3).

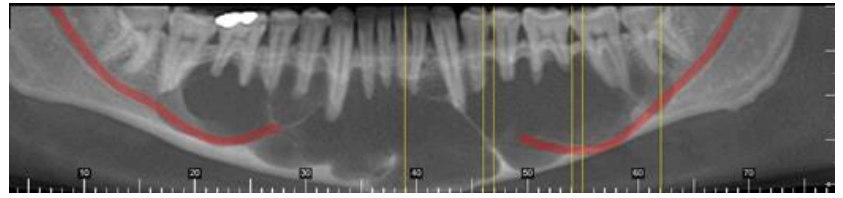

Figura 1.:Vista panorâmica da tomografia evidenciando lesão intra óssea pré-operatória.

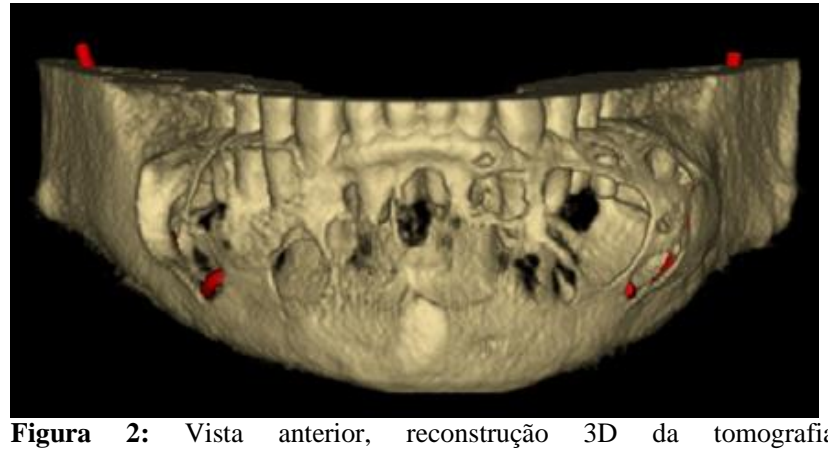
computadorizada pré-operatória.

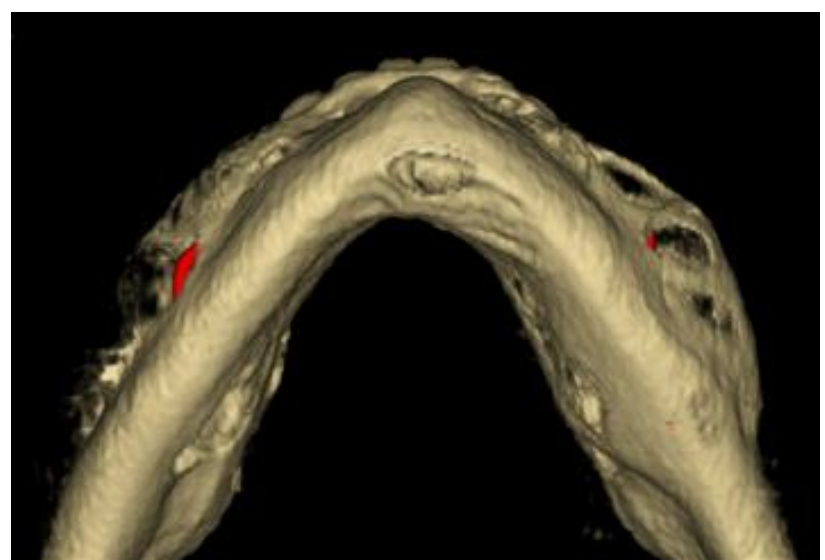

Figura 3: Vista Caldo craneana da reconstrução 3D da tomografia computadorizada pré-operatória.

À oroscopia observamos abaulamento das tabuas ósseas vestibular e lingual, ausência de mobilidade dentaria, assim como de drenagem de líquidos ou processos infecciosos. O paciente foi submetido biopsia incisional sob anestesia local, e encaminhado peça para analise histopatológica. $\mathrm{O}$ diagnóstico de ameloblastoma convencional foi confirmado.

Após confirmação paciente foi submetido curetagem sob anestesia geral. Foi realizada incisão intra oral com bisturi elétrico monopolar, estendendo- se entre os segundos molares inferiores para acesso à lesão, descolamento mucoperiosteal, acesso loja patológica, curetagem associada à osteotomia periférica com auxílio de broca esférica multilaminada sob irrigação abundante utilizando solução de soro fisiológico $0.9 \%$. Optou-se também, por realizar apicectomia dos dentes envolvidos na lesão, neurectomia dos nervos mentuais, devido intimo envolvimento com a lesão. A síntese foi realizada com fio de sutura monofilamentar de nylon 5.0. A lesão curetada foi novamente enviada para análise histopatológica, onde obtivemos a confirmação do exame realizado previamente. (Figuras 4 a 8).

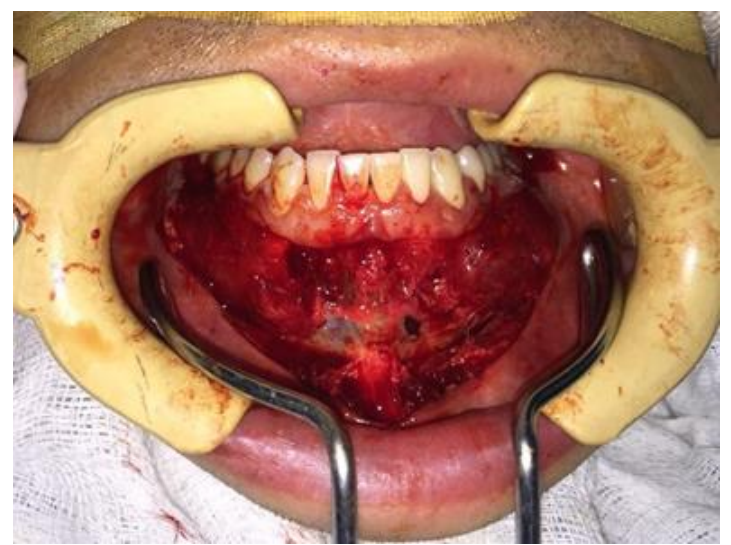

Figura 4: Acesso vestibular mandibular expondo a lesão.

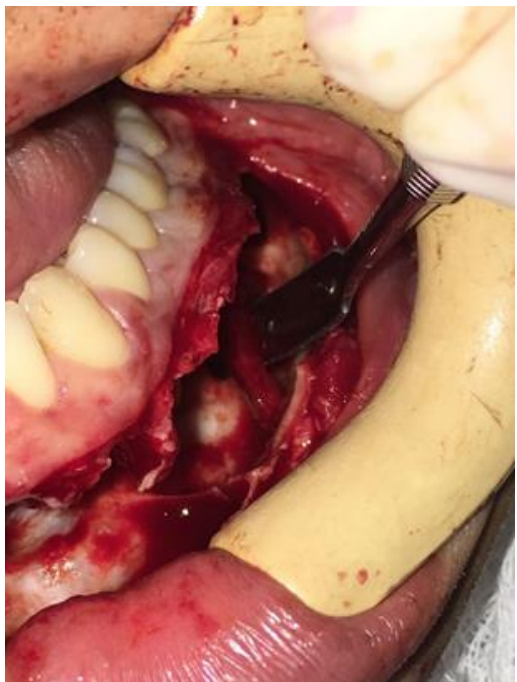

Figura 5: Feixe alveolar inferior evidenciado no trajeto mandibular após curetagem da lesão.

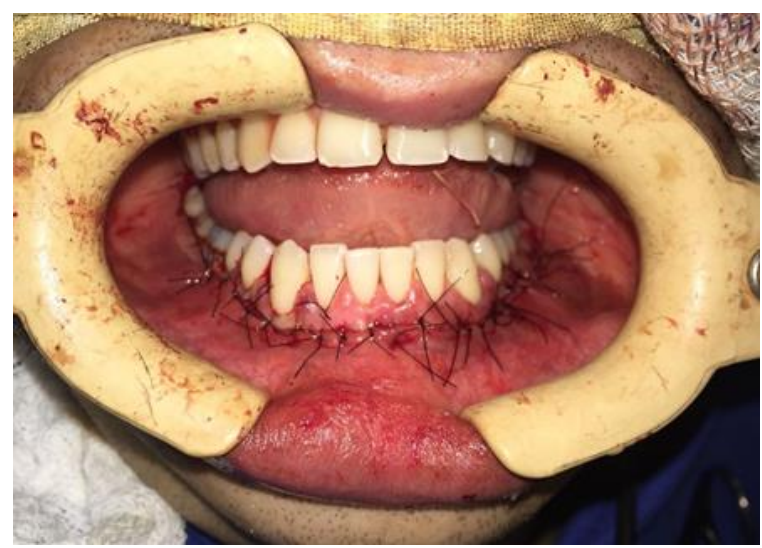

Figura 6: Aspecto final após síntese da mucosa bucal. 


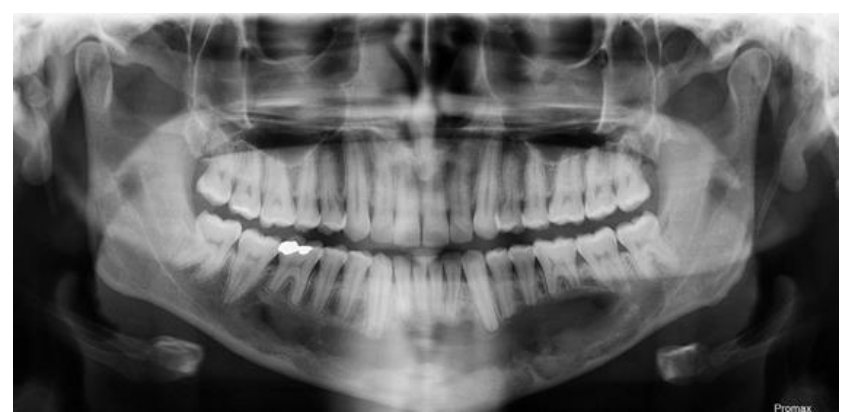

Figura 7: Ortopantomografia de controle após 13 meses.

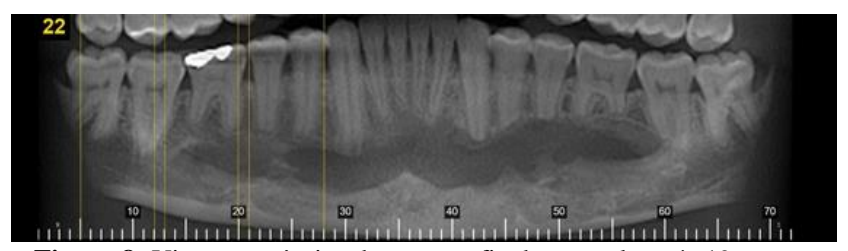

Figura 8: Vista panorâmica da tomografia de controle após 19 meses.

DISCUSSÃO

O ameloblastoma é observado em uma ampla faixa etária, não havendo consenso na literatura, demonstrando uma ampla prevalência entre a terceira e a sétima décadas de vida, não apresenta predileção por gênero ou etnia ${ }^{1,4,5}$. No presente relato, a idade do paciente diferiu do encontrado na literatura uma vez que o mesmo foi diagnostico em sua segunda década de vida. A região posterior de mandíbula é mais comumente acometida, entretanto maxila também é afetada. O tumor costuma se apresentar como um aumento de volume assintomático, com expansão das corticais ósseas, alterações de oclusão, mobilidade dentaria, porém a parestesia e dor não são tão frequentes ${ }^{1,5}$.

No exame radiográfico, tendem a se apresentar como uma imagem radiolúcida multilocular, mas podendo ser manifestar com aspectos unilocular com margens bem definidas. As lesões quando grandes, apresentam aspecto de "bolha de sabão", ou quando pequenas são relacionadas à "favos de mel"1-3. Reabsorções radiculares dos dentes adjacentes a lesão é comum, podendo estar associada terceiros molares inferiores não erupcionados ${ }^{1,2}$.

O ameloblastoma tem sido tratado de diferentes maneiras, dependendo das características clinicas, e radiográficas, levando em consideração a tendência de infiltração entre as trabéculas do $\mathrm{osso}^{\mathbf{1 3}}$. $\mathrm{O}$ uso da marsupialização apresenta desvantagens pois enquanto aguarda-se a redução do tamanho da lesão, ocorre uma continuidade da infiltração, podendo haver transformação maligna dos restos tumorais ${ }^{4}$. A enucleação seguida de curetagem da lesão consiste em uma modalidade conservadora, apesar de as vezes deixar restos de ilhas tumorais, podendo apresentar taxas de recidiva de até $80 \% 1,2,4$. Técnicas coadjuvantes como aplicação solução de Carnoy, crioterapia e ou osteotomia periférica, apresentam o intuito de reduzir taxas de recidiva ${ }^{5}$.

A ressecção óssea com margem de segurança de 1 a $2 \mathrm{~cm}$, consiste em um formato de tratamento indicado para lesões de perfil agressivo, invasivo, de grandes extensões e recorrentes ${ }^{1,5-7}$. As recidivas do ameloblastoma no tratamento radical ficam entre 15 a $38^{\% 1,7}$. A modalidade de tratamento de escolha também deve ser analisada para cada paciente individual, avaliando o tamanho, localização, comobirdades pós cirurgicas e experiencia do profissional que vai conduzir o caso $^{1,8}$. Foi optado pelo tratamento conservador inicial devido idade, localização, tamanho da lesão e possíveis sequelas pós-operatória associadas ressecções com margem.

A recidiva pode demorar anos para apresentar manifestações clinicas e radiográficas, acompanhamento longo durante período de uma década é necessário ${ }^{1}$. A transformação maligna é rara, ocorre em aproximadamente $1 \%$ dos ameloblastoma. O carcinoma ameloblástico pode surgir de um tumor primário ou de recidiva, com extensa faixa etária de ocorrência, mas com média na terceira década de vida. Frequentemente na mandíbula, associado à dor, parestesia, trismo, disfonia, ulceras e destruição óssea ${ }^{1,9,10}$. As metástases são geralmente no pulmão, seguida de linfonodos cervicais, associado à disseminação para outros ossos e órgaõ s, ${ }^{1,9}$.

\section{CONCLUSÃO}

$\mathrm{O}$ tratamento proposto se demonstrou satisfatório até o momento, visto que obtivemos neoformação óssea e foi possível evitar mutilações, deformidades ou disfunções do aparelho estomatognático geralmente causados pelo tratamento radical. Entretanto se faz necessário um maior prazo de acompanhamento para concluir eficácia total e ausência de recidiva da lesão.

\section{REFERÊNCIAS}

1. Neville BW, Allen CM, Damm DD, Chi AC. Patologia oral e maxilofacial. 4.ed. Rio de Janeiro: Guanabara Koogan; 2016.

2. Brown NA, Betz BL. Ameloblastoma: a review of recent molecular pathogenetic discoveries. Biomark Cancer. 2015;7(Suppl 2):19-24.

3. Kim SW, Jee YJ, Lee DW, Kim HK. Conservative surgical treatment for ameloblastoma: a report of three cases. J Korean Assoc Oral Maxillofac Surg. 2018;44(5):242-47.

4. Slusarenko da Silva Y, Tartaroti NA, Sendyk DI, Deboni MCZ, Naclério-Homem MG. Is conservative surgery a better choice for the solid/multicystic ameloblastoma than radical surgery regarding recurrence? A systematic review. Oral Maxillofac Surg. 2018;22(4):349-56.

5. Aramanadka C, Kamath AT, Kudva A. Recurrent Ameloblastoma: A Surgical Challenge. Case Rep Dent. 2018;2018:8271205.

6. Neagu D, Escuder-de la Torre O, Vázquez-Mahía I, Carral-Roura N, Rubín-Roger G, PenedoVázquez A et al. Surgical management of 
ameloblastoma. Review of literature. J Clin Exp Dent. 2019 Jan 1;11(1):e70-75.

7. Sharma Lamichhane N, Liu Q, Sun H, Zhang W. A case report on desmoplasticameloblastoma of anterior mandible. BMC Res Notes. 2016. doi:10.1186/s13104-016-1961-2

8. Sham E, Leong J, Maher R, Schenberg M, Leung M, Mansour AK. Mandibularameloblastoma: clinical experience and literature review. ANZ J Surg. 2009;79(10):739-44.

9. Kodati S, Majumdar S, Uppala D, Namana M. Ameloblastic carcinoma: a report of three cases. $\mathrm{J}$ Clin Diagn Res. 2016;10(10):ZD23-25.

10.Rais R, El-Mofty SK. Malignant transformation of a desmoplastic ameloblastoma to squamous cell carcinoma: a case report. Head Neck Pathol. 2019;13(4):705-10.

\section{CONFLITO DE INTERESSES}

Os autores declaram não haver conflitos de interesse.

\section{AUTOR PARA CORRESPONDENCIA}

\section{Maylson Alves Nogueira Barros}

maylson.bucomaxilofacial@gmail.com

Submetido em 12/03/2019

Aceito em 11/06/2019 